\begin{tabular}{|}
$\begin{array}{c}\text { A multilevel algorithm to compute } \\
\text { steady states of lattice Boltzmann } \\
\text { models }\end{array}$ \\
Giovanni Samaey \\
Christophe Vandekerckhove \\
Wim Vanroose \\
Report TW 560 , January 2010 \\
\hline
\end{tabular}

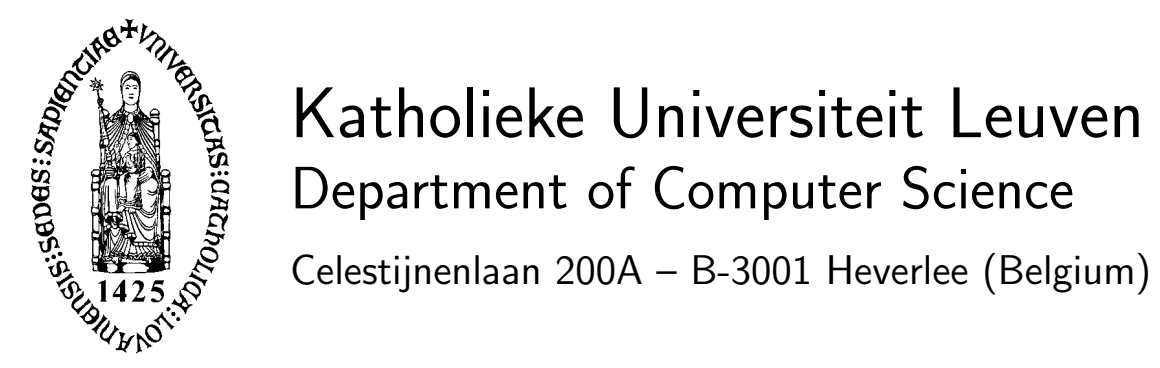




\title{
A multilevel algorithm to compute steady states of lattice Boltzmann models
}

\author{
Giovanni Samaey \\ Christophe Vandekerckhove \\ Wim Vanroose* \\ Report TW560, January 2010
}

Department of Computer Science, K.U.Leuven

\begin{abstract}
We present a multilevel algorithm to compute steady states of lattice Boltzmann models directly as fixed points of a time-stepper. At the fine scale, we use a Richardson iteration for the fixed point equation, which amounts to time-stepping towards equilibrium. This fine-scale iteration is accelerated by transferring the error to a coarse level. At this coarse level, one directly solves for the density (the zeroth moment of the lattice Boltzmann distributions), for which a coarse-level equation is known in some appropriate limit. The algorithm closely resembles the classical multigrid algorithm in spirit, structure and convergence behaviour. In this paper, we discuss the formulation of this algorithm. We give an intuitive explanation of its convergence behaviour and illustrate with numerical experiments.
\end{abstract}

* Department of Mathematics and Computer Science, Universiteit Antwerpen, Middelheimlaan 1, 2020 Antwerpen, Belgium 


\title{
A multilevel algorithm to compute steady states of lattice Boltzmann models
}

\author{
Giovanni Samaey $^{1, \star}$, Christophe Vandekerckhove ${ }^{1}$, and Wim Vanroose ${ }^{2}$ \\ 1 Scientific Computing, Department of Computer Science, K.U. Leuven, \\ Celestijnenlaan 200A, 3001 Leuven, Belgium. \\ 2 Department of Mathematics and Computer Science, Universiteit Antwerpen, \\ Middelheimlaan 1, 2020 Antwerpen, Belgium.
}

\begin{abstract}
We present a multilevel algorithm to compute steady states of lattice Boltzmann models directly as fixed points of a time-stepper. At the fine scale, we use a Richardson iteration for the fixed point equation, which amounts to time-stepping towards equilibrium. This fine-scale iteration is accelerated by transferring the error to a coarse level. At this coarse level, one directly solves for the density (the zeroth moment of the lattice Boltzmann distributions), for which a coarse-level equation is known in some appropriate limit. The algorithm closely resembles the classical multigrid algorithm in spirit, structure and convergence behaviour. In this paper, we discuss the formulation of this algorithm. We give an intuitive explanation of its convergence behaviour and illustrate with numerical experiments.
\end{abstract}

\section{Introduction}

For a broad class of systems, in applications ranging from physics, fluid flow $[10,9]$ and biology [2] to traffic flow [4], a macroscopic (coarse) partial differential equation (PDE) that models density evolution in a space-time domain, is often insufficient to accurately describe physical interactions between individual particles (atoms, molecules, bacteria, vehicles). For instance, the dynamics of a system of colliding particles with interactions that depend sensitively on the relative particle velocities can, in general, not be modeled completely by a reaction-diffusion equation for the particle density [13]. In such cases, one needs to resort to a more microscopic (fine-scale) description, such as a kinetic equation that models the evolution of the velocity-position phase space distribution density of particles as a combination of advection according the the current velocity and collisions that redistribute velocities [25].

A lattice Boltzmann model (LBM) is a simple and effective space-timevelocity discretization of a fine-scale kinetic equation [20]. Only a low number of velocities are considered, and the discrete velocities are related to the lattice spacing and time step in such a way that the corresponding distributions are simply shifted over an integer number of lattice sites over one time step. The

\footnotetext{
* GS is a Postdoctoral Fellow of the Research Foundation - Flanders (FWO Vlaanderen).
} 
advantage is that one can decompose evolution in two separated steps: first, one simply advects each distribution function according to its corresponding velocity; subsequently, one performs a collision step in which the velocities are redistributed. A more precise mathematical description of LBMs will be presented in section 2 .

In the appropriate diffusion limit [15, e.g.], the above-described fine-scale kinetic models collapse onto a limiting coarse partial differential equation. However, their associated computational cost is much higher, due to the increased dimension. For such cases, several approaches have been developed that exploit the link between the coarse and fine-scale levels of descriptions to significantly accelerate computations. Of particular importance in this work is the equation-free framework that was proposed by Kevrekidis et al. [11,21]. The framework introduces lifting and restriction operators to map a coarse state to a fine-scale state and vice versa. These operators are then used to construct a coarse time-stepper for the unavailable coarse equation as a three step procedure: (1) lifting, i.e. the creation of appropriate initial conditions for the fine-scale model, conditioned upon the coarse state at time $t^{*} ;(2)$ simulation, using the fine-scale model, over the time interval $\left[t^{*}, t^{*}+\Delta t\right]$; and (3) restriction, i.e. the extraction of the coarse state at time $t^{*}+\Delta t$. The resulting coarse time- $\Delta t$ map can then be used in conjunction with projective integration methods [7] to accelerate time integration, or with a matrix-free algorithm to directly compute coarse steady states [17-19]. We refer to [12] for a recent review and references to related methods.

In this paper, we borrow important concepts from the equation-free framework, and use them to construct a new algorithm that directly computes fine-scale steady states of an LBM as fixed points of the time-stepper. The proposed algorithm bears many similarities with standard multigrid [1,22]. Multigrid is a sophisticated iterative method for the computation of a steady state of a PDE. It is most easily understood by realizing that the error in any initial guess consists of high and low wavenumber modes. Basic iterative methods (such as a Jacobi or Gauss-Seidel iteration, or Richardson's method, which corresponds to time-stepping) are very efficient in removing highfrequency error components; such methods are therefore called smoothers. In multigrid, one uses the fact that the smoother yields errors (and residuals) that can be accurately represented on a coarser mesh (since they are smooth). One then transfers the residuals to the coarser grid (restriction) and solves for the error. The resulting error is then transferred back to the finer grid (prolongation). This sequence is called coarse grid correction, and the combination of smoothing and coarse grid correction yields a very powerful algorithm. At the the coarse grid, the same procedure can be applied. One then performs again a few smoothing steps and a coarse grid correction on an even coarser grid, leading to a hierarchy of grids - hence the name multigrid.

The multilevel algorithm that we propose here follow the same strategy. At the fine scale, we consider the computation of a fixed point of a lattice Boltzmann time-stepper; the basic iteration (smoother) on this level is a 
Richardson iteration, which amounts to time-stepping towards equilibrium. Instead of moving to a coarser grid, as in multigrid, however, here we move to a coarser level of description, i.e. the level of the coarse PDE. Note that at this level, we can only use the PDE that is known in the diffusion limit, which yields only an approximation to the evolution of the density of the LBM. The transfer operators that connect the coarse and fine-scale levels of description are exactly the lifting and restriction operators that have been introduced in the equation-free framework; these then replace the prolongation and restriction operators that are used in multigrid.

The remainder of the paper is organized as follows. In section 2 we define our model problems. Section 3 describes the behaviour of Richardson iteration for this problem. We then proceed to formulate our multilevel algorithm in section 4. Section 5 contains an intuitive explanation of the convergence behaviour. We conclude in section 6 .

\section{Model problem}

\subsection{Lattice Boltzmann models}

Throughout this paper, we consider reaction-diffusion lattice Boltzmann models (LBMs) $[3,20]$ in one space dimension as the illustrative example. We briefly review the principles of LBMs and describe the specific model problem of this paper.

We define a discrete number of particle distribution functions $f_{i}\left(x_{j}, t_{n}\right)$, with $1 \leq j \leq N,-q \leq i \leq q$ and $n \geq 0$, with grid spacing $\delta x$ in space and $\delta t$ in time, and choose the velocities $v_{i}$ such that they correspond to a movement over an integer number of lattice points during one time-step,

$$
v_{i}=c_{i} \frac{\delta x}{\delta t}, \quad c_{i}=-q, \ldots,-1,0,1, \ldots, q .
$$

Furthermore, we introduce equilibrium distributions $f_{i}^{e q}(x, t)=w_{i}^{e q} \rho(x, t)$, which correspond to local diffusive equilibrium. Here, the coefficients $w_{i}^{e q}=$ $w_{-i}^{e q}$ depend on the number of speeds $2 q+1$.

Remark 1 (Notation). To avoid potential confusion, we emphasize that, in this text, we will use the symbol $\delta t$ (and, for notational consistency, $\delta x$ ) to denote the parameters of the fine-scale (lattice Boltzmann) time-stepper, whereas the symbol $\Delta t$ is used to indicate the size of a coarse time step.

With these definitions, we can write an evolution law for the distributions $f(x, t)=\left(f_{i}(x, t)\right)_{i=-q}^{q}$ as

$f_{i}\left(x+c_{i} \delta x, t+\delta t\right)=(1-\omega) f_{i}(x, t)-\omega f_{i}^{e q}(x, t)+\beta \sum_{j} V_{i j} f_{j}(x, t)+R_{i}(x, t)$, 
which we denote in compact form as

$$
\mathbf{f}^{n+1}=\phi\left(\mathbf{f}^{n}\right),
$$

where $\mathbf{f}=\left(f_{i}(\mathbf{x})\right)_{i=-q}^{q}, \mathbf{x}=\left(x_{j}\right)_{j=1}^{N}$ and the superscript $n$ denotes the time instance $t=n \delta t$. Equation (1) decomposes evolution into a collision phase (the right-hand side) and a streaming phase, during which the post-collision values are propagated to a neighboring site. The first two terms of the righthand side represent a BGK relaxation to local diffusive equilibrium on a characteristic time scale $\tau=1 / \omega$. The third term is an external force, which is discretized as proposed by Luo [14], and the terms $R_{i}(x, t)$ model reactive collisions. Note that, for pure diffusion, a lattice Boltzmann time-stepper is stable for $\omega \in[0,2]$.

We now define the first few (non-dimensional) moments of the distribution function as

$\rho(x, t)=\sum_{i=-q}^{q} f_{i}(x, t), \quad \varphi(x, t)=\sum_{i=-q}^{q} c_{i} f_{i}(x, t), \quad \xi(x, t)=\frac{1}{2} \sum_{i=-q}^{q} c_{i}^{2} f_{i}(x, t)$,

which represent density, momentum and energy, respectively. The lattice Boltzmann equation can be written equivalently as a set of coupled PDEs for the $2 q+1$ moments of the individual distribution functions. A ChapmanEnskog expansion shows that, when the density is sufficiently smooth, the lattice Boltzmann model (1) (or the set of coupled equations for the moments) can be approximated by a single reaction-advection-diffusion equation only depending on the density $[5,16]$,

$\partial_{t} \rho(x, t)=\partial_{x}\left(D \partial_{x} \rho(x, t)\right)+\beta \partial_{x} \rho(x, t)+r(\rho(x, t)), \quad D=\frac{2-\omega}{2 \omega} \frac{\delta x^{2}}{\delta t} \sum_{i} c_{i}^{2} w_{i}^{e q}$,

where the transport coefficients $D$ and $\beta$ and the reaction rate $r(\rho)$ depend on the relaxation, external force and collision terms in the lattice Boltzmann model. When the reaction terms $R_{i}$ can be written as functions of the density, $R_{i}(x, t)=w_{i}^{e q} \delta t R(\rho(x, t))$, we obtain $r(\rho)=R(\rho)$. In general, however, if $R_{i}(x, t)$ depend on the individual distribution functions, it is cumbersome to eliminate the velocity dependence and derive an explicit formula for $r(\rho)$.

From the assumption that a coarse model for $\rho(x, t)$ exists, it follows that the higher order moments $\varphi(x, t)$ and $\xi(x, t)$ can be written as functionals of $\rho(x, t)$. (We call this slaving.) For a 3 -speed lattice Boltzmann reactiondiffusion model, i.e. equation (1) with $q=1$ and $\beta=0$, these slaving relations can be written down analytically as an asymptotic expansion in $1 / \omega$. Up to 
third order, we have [23],

$$
\begin{aligned}
& \varphi(x, t)=-\frac{2 \delta x}{3 \omega} \partial_{x} \rho+\frac{\delta x \delta t}{3 \omega^{2}} \frac{(4 \omega-2)}{(\omega-2)} \partial_{x} r(\rho)+\frac{\delta x \delta t}{3 \omega^{2}} \frac{\left(-2 \omega^{2}+2 \omega-2\right)}{(\omega-2)} \partial_{x t}^{2} \rho(x, t) \\
& \xi(x, t)=\frac{1}{3} \rho(x, t)-\frac{\delta t}{6 \omega}\left(r(\rho(x, t))-\partial_{t} \rho(x, t)\right) .
\end{aligned}
$$

These expansions can alternatively be written down in terms of $\rho(x, t)$ and its spatial derivatives only, by making use of (4).

\subsection{Fixed point formulation}

We are interested in the fixed points of equation (2), i.e. solutions of the nonlinear equation

$$
\mathbf{f}^{*}-\phi\left(\mathbf{f}^{*}\right)=0
$$

This nonlinear problem can be solved using Newton's method. We will apply a multilevel algorithm on the linear systems arising in each Newton iteration, which can be written as

$$
\left(I-J_{\phi}(\overline{\mathbf{f}})\right) \Delta \mathbf{f}=-r(\overline{\mathbf{f}})
$$

or, for short,

$$
A_{f} \mathbf{x}=b .
$$

Here, we use $\mathbf{x}=\Delta \mathbf{f}$ and $J_{\phi}(\overline{\mathbf{f}})=(\partial \phi / \partial \mathbf{f})(\overline{\mathbf{f}})$ and $r(\overline{\mathbf{f}})=\overline{\mathbf{f}}-\phi(\overline{\mathbf{f}})$ denote the Jacobian of $\phi$ and the residual of equation (6), respectively, evaluated at a current guess $\overline{\mathbf{f}}$. For notational convenience, we have eliminated the dependence on $\overline{\mathbf{f}}$ in $A_{f}$ and $b$. The Jacobian $J_{\phi}$ is not available in closed form, since we can only evaluate the time-stepper, but Jacobian-vector products can be estimated as

$$
J_{\phi}(\overline{\mathbf{f}}) \mathbf{v} \approx \frac{\phi(\overline{\mathbf{f}}+\epsilon \mathbf{v})-\phi(\overline{\mathbf{f}})}{\epsilon},
$$

with $\epsilon$ appropriately small. This enables the use of methods that require only matrix-vector products.

\section{Richardson iteration}

We first consider a basic iterative method, namely Richardson iteration,

$$
\mathbf{x}^{(k+1)}=S\left(\mathbf{x}^{(k)}, A_{f}, b\right)=\mathbf{x}^{(k)}+\left(A_{f} \mathbf{x}^{(k)}-b\right),
$$

which is selected over alternatives such as Jacobi or Gauss-Seidel, because it does not involve a matrix splitting of $A_{f}$, and hence requires only matrixvector products. This method can be seen to correspond to time-stepping for 
the error equation of the linearized system. To see this, we elaborate

$$
\begin{aligned}
\mathbf{x}^{(k+1)} & =\mathbf{x}^{(k)}+\left(A_{f} \mathbf{x}^{(k)}-b\right) \\
& =\mathbf{x}^{(k)}+\left(\left(I-J_{\phi}\right) \mathbf{x}^{(k)}-b\right) \\
& =J_{\phi} \mathbf{x}^{(k)}-b .
\end{aligned}
$$

The last equation shows that the Richardson iteration corresponds to timestepping with the linearized time-stepper, modified by the righthand side of the linear system (the residual of the nonlinear equation). Hence, the spectral properties of the smoothing operator will be those of the linearized timestepper.

Recall that we assume that an equation exists that closes at the level of the density $\rho(x, t)$ alone. As a consequence, this assumption implies that the higher order moments become functionals of $\rho(x, t)$ on time-scales which are fast compared to the overall system evolution (slaving). This behaviour is reflected in the spectral properties of the smoother: Richardson iterations quickly reduce errors in the higher order moments, whereas errors in modes that correspond to the density are not significantly affected by the smoother. This observation is illustrated by a numerical experiment.

We consider the pure diffusion 3-speed lattice Boltzmann problem, i.e. equation (1) with $q=1, \beta=0$ and $R_{i} \equiv 0$ using periodic boundary conditions. The domain is $[0,1]$, and the model parameters are chosen to be $D=1$, $\omega=1.25$ and $\delta x=1 / 128$. The time step then follows from equation (4). A fixed point of this time-stepper can be found as the solution of the linear system

$$
A_{f} \mathbf{x}=0 .
$$

We now look into the behaviour of Richardson iteration for the linear system (10).

Remark 2. Because of the periodic boundary conditions, the time-stepper has a trivial eigenvalue 1, and consequently, the fixed point problem is singular. This singularity can be removed by adding a phase condition and an artificial parameter, see e.g. [19]. In this experiment, we perform a spectral analysis of Richardson iteration; for this purpose, simply considering Richardson iteration on the singular system is sufficient.

Because of the homogeneous righthand side, Richardson iterations reduce to time-stepping with the original linear time-stepper. For the 3 -speed model, there is a linear 1-to-1 relation between the distribution functions and the moments (3). It can easily be verified (for instance using Maple) that, when working in the equivalent moment description, the eigenvectors have the form $(\rho, \varphi, \xi)=(A \sin (k \cdot x), B \cos (k \cdot x), C \sin (k \cdot x))$, with $1 \leq k \leq N-1$. For each frequency, there are 3 eigenmodes. However, the analytic determination of the constants, as well as the corresponding eigenvalues, does not yield a workable closed formula. Therefore, we compute the spectrum numerically. 

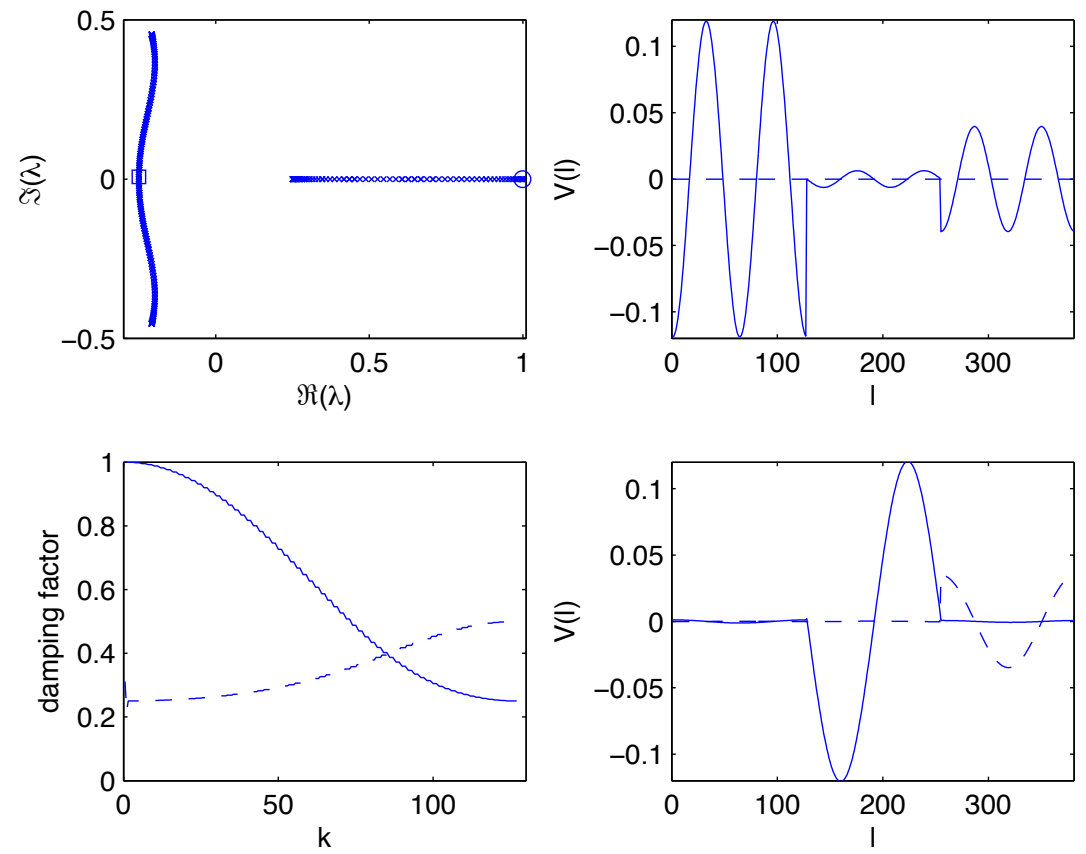

Fig. 1. Top left: Spectrum of the lattice Boltzmann time-stepper/Richardson smoother for homogeneous diffusion. Bottom left: Richardson amplification factors for each of the eigenmodes, as a function of wave number $k$. The solid line corresponds to the amplifications factors of the real positive eigenvalues; the dashed line corresponds to the amplifications factors of the complex eigenvalues with negative real part. Right: a typical eigenmode corresponding to a real positive eigenvalue (top) and a complex eigenvalue (bottom) of the time-stepper. The solid line contains the real part; the dashed line is the imaginary part. The corresponding eigenvalues are depicted by a square, resp. circle in the top left panel. Eigenmodes are displayed in moment representation: the leftmost part of each eigenvector corresponds to density, the middle part to momentum and the rightmost part to energy. 
The results are depicted in figure 1 (top left). It is seen that the spectrum breaks down into two sets of eigenvalues: a set of $N-1$ real and positive eigenvalues, and a set of $2 N-2$ complex eigenvalues with negative real part. Figure 1 (right) shows an eigenvector corresponding to a particular mode in each of these two sets, in the moment representation. One can visually verify that the eigenvectors corresponding to the rightmost set of real eigenvalues appear to satisfy the slaving relations (5); we call these the slaved modes. The slaving relations are not satified for the eigenvectors corresponding to the set of complex eigenvalues (non-slaved modes). Clearly, the slower a mode is damped, the better the slaving relations are satisfied.m

We now look at the behaviour of the Richardson iteration for each of these eigenmodes individually. Starting from a normalized eigenmode that satisfies $\max _{l} V_{l}=1$ (with $1 \leq l \leq 3 N-3$ denoting the components), we perform a single Richardson iteration and look at the amplification factor. Figure 1 (bottom left) shows the maximal value of the resulting vector as a function of the wave number. As could be expected from the spectrum of the time-stepper, we can draw two main conclusions :

- Regardless of the wave number, modes that corresponds to non-slaved states are damped quickly by the time-stepper; only modes that corresponds to slaved states can persist over longer time scales.

- For the slaved modes, we observe that low wavenumber modes are damped much more slowly than high wavenumber waves.

Based on these observations, we conclude that it would be beneficial to combine Richardson iterations with a method to directly reduce the errors in the density. To this end, we now turn to a multilevel algorithm.

\section{Multilevel algorithm}

To solve the linear system (8) more efficiently, we make use of the link between the fine-scale model (1) and the approximate coarse description (4). We first describe lifting and restriction operators that map coarse to fine-scale states, and vice versa. We then proceed to formulate the complete algorithm. An intuitive explanation on it convergence properties is deferred to section 5 .

\subsection{Lifting and restriction}

We consider operators that define a mapping between the distribution functions $f_{i}(x, t)$ and the density $\rho(x, t)$. A restriction operator, mapping the distribution $f(x, t)=\left(f_{i}(x, t)\right)_{i}$ to the density $\rho(x, t)$,

$$
\mathcal{R}: f(x, t) \mapsto \rho(x, t)=\mathcal{R}(f(x, t)),
$$

can easily be obtained using (3). The converse lifting operator, reconstructing distributions from the density,

$$
\mathcal{L}: \rho(x, t) \mapsto f(x, t)=\mathcal{L}(\rho(x, t)),
$$


is considerably more involved.

One could simply initialize the distributions as

$$
f_{i}(x, t)=w_{i} \rho(x, t), \quad \sum_{i} w_{i}=1,
$$

where $w_{i}=w_{i}^{e q}$ is an obvious choice, since this corresponds to the local diffusive equilibrium. Via a Chapman-Enskog expansion, it can be shown that this corresponds to a zeroth order approximation of the slaving relations [23]. Clearly, initializing also the higher order terms in the Chapman-Enskog expansion yields a more accurate reconstruction.

A numerical procedure to obtain a second order approximation to the slaving relations, using only the time-stepper (2) is the constrained runs scheme $[8,6]$. Here, during a preparatory step, a number of lattice Boltzmann timesteps is performed, after each of which the density is reset. This scheme can be shown to converge linearly, with a convergence factor $|1-\omega|$ [23]; hence, it is also stable whenever $\omega \in[0,2]$. Higher order variants, which are more accurate but may become unstable, have been investigated in [24].

\subsection{Coarse-level correction}

Once the fine-scale state has been transferred to the coarse level, we need to define the coarse linear system that needs to be solved. For this, we start from the approximate coarse model (4), and construct a corresponding timestepper, which we write in compact form as

$$
\rho^{n+1}=\Phi\left(\rho^{n}\right),
$$

where $\rho=\rho(\mathbf{x})$.

The fixed-point equation at this level can then equivalently be written as

$$
\left(I-J_{\Phi}(\bar{\rho})\right) \Delta \rho=-R(\bar{\rho}),
$$

which we write in a shorthand notation

$$
A_{c} \mathbf{X}=B
$$

Here, we use $\mathbf{X}=\Delta \rho$, and $J_{\Phi}(\bar{\rho})=(\partial \Phi / \partial \rho)(\bar{\rho})$ and $R(\bar{\rho})=\bar{\rho}-\Phi(\bar{\rho})$ denote the Jacobian of $\Phi$ and the residual of the coarse fixed-point equation, respectively, evaluated at a current guess $\bar{\rho}$.

\subsection{The multilevel algorithm}

With all building blocks in place, we are now ready to formulate the complete multilevel algorithm. One iteration, starting from $\mathbf{x}^{(m)}$, consists of the following steps: 
1. Presmoothing: Perform $\nu_{1}$ Richardson iterations,

$$
\overline{\mathbf{x}}^{(m)}=S^{\nu_{1}}\left(\mathbf{x}^{(m)}, b\right) .
$$

\section{Coarse-level correction:}

- Compute the defect: $d^{(m)}=b-A \mathbf{x}^{\overline{(m)}}$.

- Restrict defect : $\left.D^{(} m\right)=\mathcal{R}\left(d^{(m)}\right)$.

- Coarse-level solve : $A_{c} \mathbf{V}^{(m)}=D^{(m)}$.

- Lift correction : $v^{(m)}=\mathcal{L}\left(\mathbf{V}^{(m)}\right)$.

- Update fine-scale solution : $\hat{\mathbf{x}}^{(m)}=\bar{x}^{(m)}+v^{(m)}$.

3. Postsmoothing: Perform $\nu_{2}$ Richardson iterations,

$$
\mathbf{x}^{(m+1)}=S^{\nu_{2}}\left(\hat{\mathbf{x}}^{(m)}, b\right) .
$$

Remark 3 (Comparison with multigrid). The algorithmic structure resembles that of a classical multigrid method to find steady states of partial differential equations $[1,22]$. For the PDE case, the amplification factors of a coarselevel Richardson iteration are similar to the solid line in figure 1 (bottom left). Multigrid combines the good smoothing properties for high wavenumber modes with a coarse grid correction. One first performs a number of smoothing steps, after which one transfers the residuals to the coarser grid (restriction) and solves for the error. This restriction is possible, because a smooth residual can be accurately represented on a coarser mesh. The resulting error is then transferred back to the finer grid (prolongation), and added to the current guess. Here, exactly the same procedure is followed; only the prolongation and restriction have been replaced by equation-free lifting and restriction. Classical multigrid can be used for the coarse level correction.

Remark 4 (Full approximation scheme). Note that, as in multigrid, we transfer the residual to the coarse level, and solve the error equation there. Instead of using Newton's method with a multilevel algorithm to solve the linear systems in each iteration, the nonlinear equation (6) can also be solved using a nonlinear multilevel algorithm directly, in the spirit of a nonlinear multigrid method (the full approximation scheme) $[1,22]$.

\section{Convergence of the two-level cycle}

We now proceed to show how smoothing and coarse-level correction work together to yield fast convergence. To this end, we adapt the exposition that was presented in [1] for the classical multigrid method to our setting.

\subsection{An intuitive algebraic picture}

We start with an illustrative numerical example. We consider the pure diffusion 3-speed lattice Boltzmann problem, i.e. equation (1) with $q=1, \beta=0$ 
and $R_{i} \equiv 0$ using Dirichlet boundary conditions. The domain is $[0,1]$, and the model parameters are chosen to be $D=1, \omega=1.1$ and $\delta x=1 / 128$. The time step then follows from equation (4). We again look for a fixed point of the time-stepper as the solution of the linear system (10). To this end, we perform two iterations of the multilevel algorithm using $\nu_{1}=2$ presmoothing and $\nu_{2}=2$ postsmoothing steps, starting from an initial guess $\mathbf{f}$ that has the moment representation

$$
\rho(\mathbf{x})=\varphi(\mathbf{x})=\xi(\mathbf{x})=\sin (3 \mathbf{x})+\sin (45 \mathbf{x}) .
$$

The evolution of the error throughout the algorithm is depicted in figure 2. The figure shows the effect of presmoothing, coarse-level correction and postsmoothing during both iterations. We see that during the presmoothing step the error in $\phi$ and $\xi$ decreases rapidly, as well as the error in the high wavenumber modes of $\rho$. However, the low wavenumber modes of $\rho$ remain virtually unaffected by the presmoothing. In the next step, we solve for the density error at the coarse-level correction, and lift the resulting error the fine-scale representation using the zeroth-order term of the ChapmanEnskog expansion. We remark that during this lifting step, the error in $\xi$ also decreases substantially, as a consequence of the form of the slaving relations (5)). Finally, in the postsmoothing phase, the error in the higher order moments is again decreased. Note that the coarse-level equation does not correspond exactly to the behaviour of the density of the lattice Boltzmann model, and hence the resulting density error does not vanish. The lattice Boltzmann model converges to the limiting density equation for $\omega \rightarrow 1$, so the density error that remains after the coarse-level solve will be larger if $\omega$ is further away from 1 . This is illustrated in figure 3 , where the experiment is repeated with $\omega=1.25$.

We now proceed to providing an intuitive, graphical explanation of this behaviour, see figure 4 . The exposition is very similar in spirit to the intuitive picture that was given in [1, Chapter 5]. In panel (a), we show the total error $e_{f}$ in the current guess, and decompose this error according to the subspaces that correspond to slaved and non-slaved eigenmodes (the dashed coordinate system). We can also make a second decomposition that corresponds to the transition between the coarse and fine-scale levels of description. A first subspace contains the fine-scale modes that can be represented given only the density, i.e. the range of the lifting operator $\operatorname{Range}(\mathcal{L})$. Because the lifting operator can only reconstruct the fine-scale state approximately, the subspace of the slaved modes and Range $(\mathcal{L})$ do not coincide exactly. The complementary space contains all remaining fine-scale modes, i.e. the modes that map to a zero density, the null space of the restriction operator $\operatorname{Null}(\mathcal{R})$. Also this space does not correspond exactly to the slaved modes. We will now look into the behaviour of the multilevel algoritm in these decompositions. The first step in the algorithm is presmoothing, depicted in panel (b). Here, the error in non-slaved modes decrease quickly, while the error in the slaved modes is virtually unaltered. (Note from the experiment before that this is only ap- 

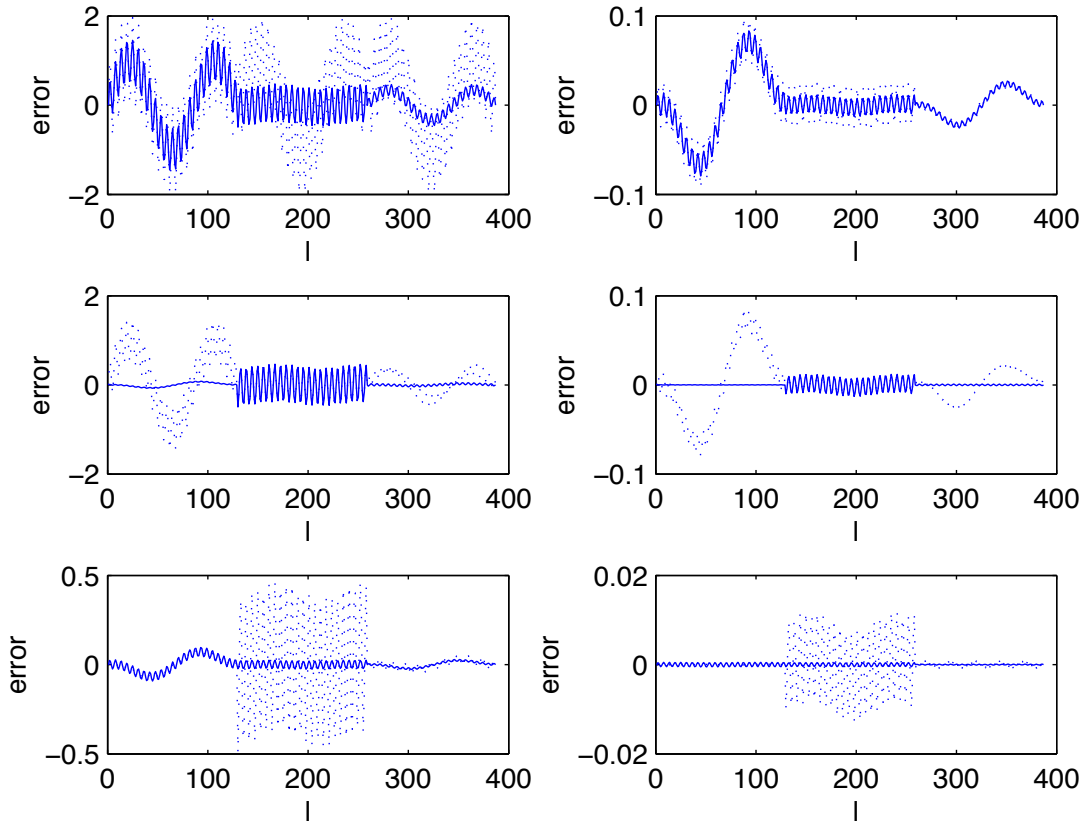

Fig. 2. Evolution of error throughout the multilevel iteration for a pure diffusion lattice Boltzmann model with $\omega=1.1$. (The other parameters are in the text.) Left: first iteration. Right: second iteration. Dashed, resp. solid, lines represent the error before, resp. after the presmoothing phase (top), the coarse-level correction (middle) and the postsmoothing phase (bottom). Eigenmodes are displayed in moment representation: the leftmost part corresponds to density, the middle part to momentum and the rightmost part to energy. 

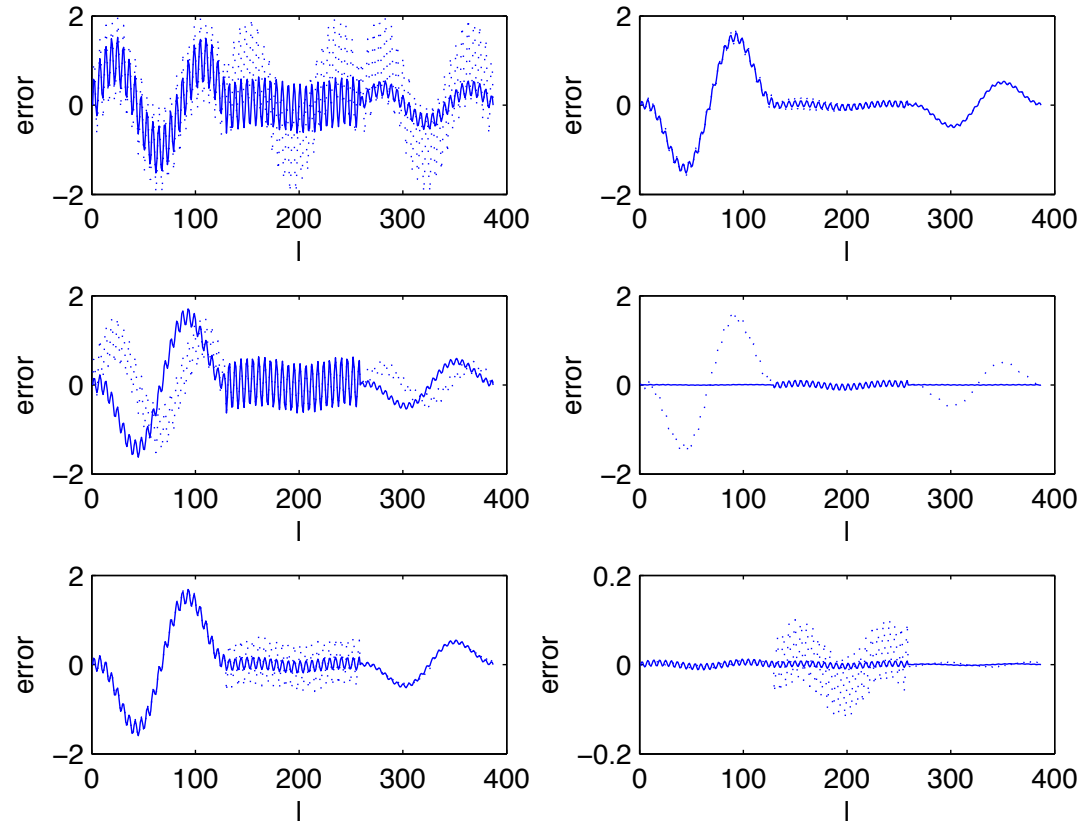

Fig. 3. Evolution of error throughout the multilevel iteration for a pure diffusion lattice Boltzmann model with $\omega=1.25$. (The other parameters are in the text.) Left: first iteration. Right: second iteration. Dashed, resp. solid, lines represent the error before, resp. after the presmoothing phase (top), the coarse-level correction (middle) and the postsmoothing phase (bottom). Eigenmodes are displayed in moment representation: the leftmost part corresponds to density, the middle part to momentum and the rightmost part to energy. 


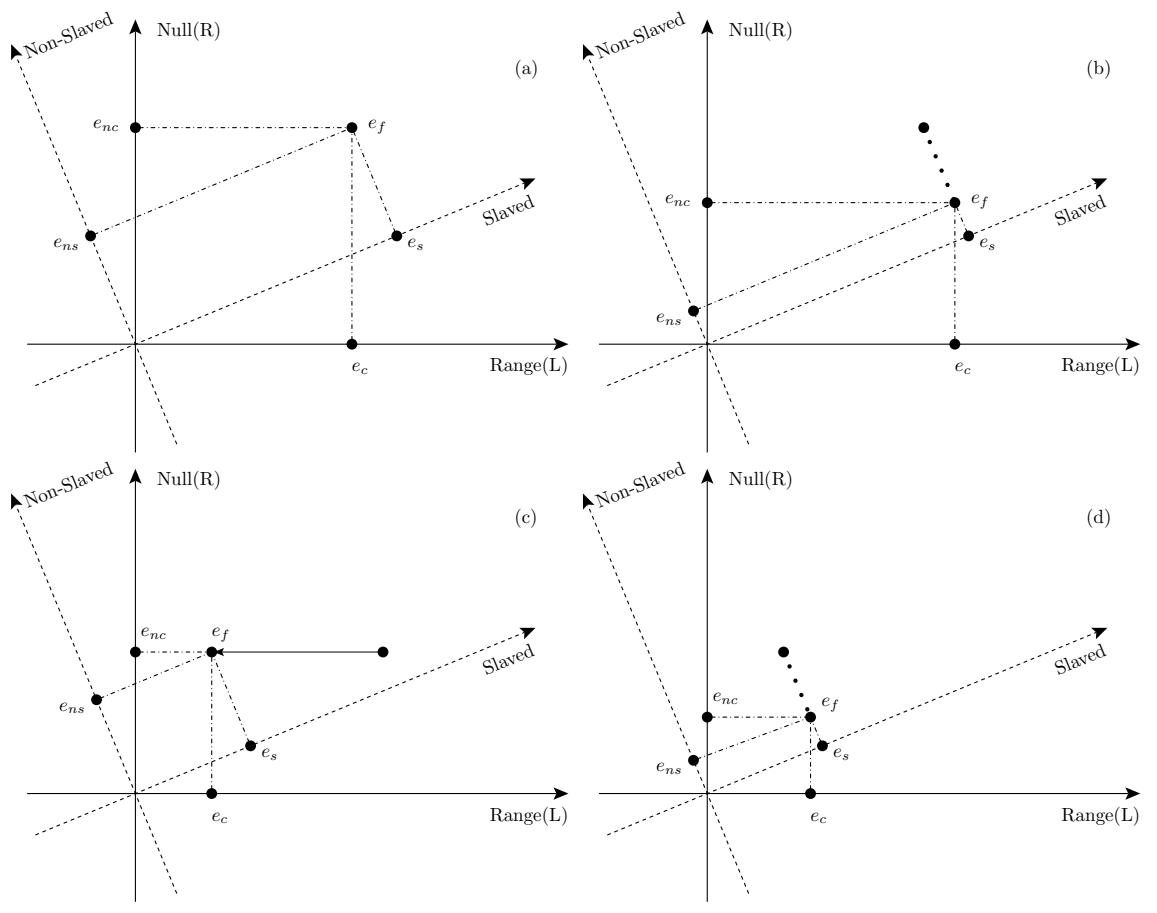

Fig. 4. Schematic representation of the error at different stages of the multilevel algorithm: (a) initial error; (b) the effect of presmoothing; (c) the effect of coarse-level correction; and (d) the effect of postsmoothing. Shown is how the error decomposes in terms of (i) the range of the lifting operator and the nullspace of restriction; and (ii) slaved and non-slaved eigenmodes of the lattice Boltzmann time-stepper. 
proximately true, and only for the low wavenumber components.) Next, we transfer the error to the coarse level and perform a coarse-level solve, see panel (c). In this phase, the part of the error that is in $\operatorname{Range}(\mathcal{L})$ is significantly reduced. Due to the fact that the density equation only approximates the behaviour of the density of the lattice Boltzmann model, this part of the error is not put exactly back to zero. We also observe that, in this step, the error in the non-slaved modes has increased again. This is due to the lifting operator, which reconstructs the fine-scale state based on the density alone, reintroducing artifacts. In a final postsmoothing step, see panel (d), these non-slaved modes are again removed by a number of additional smoothing steps.

\subsection{Numerical convergence tests}

Let us now proceed to numerically illustrate convergence. We again consider the pure diffusion 3-speed lattice Boltzmann problem, i.e. equation (1) with $q=1, \beta=0$ and $R_{i} \equiv 0$ using Dirichlet boundary conditions. The domain is $[0,1]$, and the model parameters are chosen to be $D=1, \delta x=1 / 128$, and $\omega=1.1$, resp. $\omega=1.25$. The time step then follows from equation (4). We again look for a fixed point of the time-stepper as the solution of the linear system (10) using the multilevel algorithm with $\nu_{1}=2$ presmoothing and

$\nu_{2}=2$ postsmoothing steps, starting from a random initial guess. Figure 5 shows the two-norm of the error as a function of the iteration number. We see linear convergence, with a convergence rate that depends on $\omega$. As follows from the algebraic picture above, this can be explained by noting that the coarse-level correction reduces more error in the density when $\omega$ is closer to 1 . We verified that the convergence rates are mesh-independent.

\section{Conclusions}

We presented a multilevel algorithm that accelerates the iterative computation of steady states of lattice Boltzmann models. The algorithm exploits the link between a fine-scale description and a coarse-scale limiting equation. As in multigrid, the error is decomposed into fine-scale and coarse components, which are each handled independently. In multigrid, the fine and coarse scales correspond to high and low wavenumber modes. In our algorithm, the error is decomposed into a coarse-scale component, which is defined by a reduced description in terms of density alone, and the remaining fine-scale components. The fine-scale component of the error is quickly damped by straightforward time integration; the coarse component, on the other hand, is reduced via the solution of an approximate coarse-scale equation.

The algorithm relies on the definition of appropriate lifting and restriction operators to transfer the error from the coarse to the fine level and vice versa. Such operators have been developed in the equation-free framework $[11,12]$ 


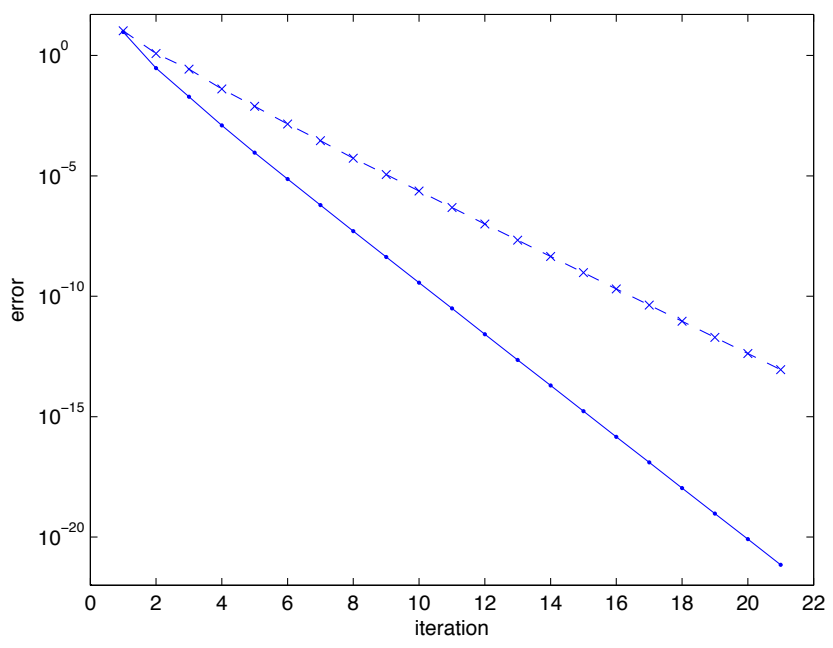

Fig. 5. Two-norm of the error of the multilevel iteration as a function of the iteration number for a fixed point computation of a lattice Boltzmann time-stepper with $\omega=1.1$ (solid) and $\omega=1.25$ (dashed), starting from a random initial guess.

for a variety of fine-scale systems. In cases where fine-scale steady states exist (such as the kinetic formulations in this paper), our algorithm can provide an efficient means to compute these fixed points. We note that, on the coarse level, one could also use a multigrid algorithm for the solution of the coarse level system, resulting in a method with overall linear complexity, also in higher spatial dimension.

In future work, we will extend this work to more general discretization of kinetic equations, and perform a more rigourous two-grid analysis.

\section{Acknowledgements}

This work was supported by the Research Foundation - Flanders through Research Projects G.0130.03 and G.0170.08 and by the Interuniversity Attraction Poles Programme of the Belgian Science Policy Office through grant IUAP/V/22 (GS). The scientific responsibility rests with its authors.

\section{References}

1. W Briggs, VE Henson, and S McCormick. A multigrid tutorial. SIAM, 2000.

2. FACC Chalub, PA Markowich, B Perthame, and Christian Schmeiser. Kinetic models for chemotaxis and their drift-diffusion limits. Monatsh Math, 142(12):123-141, 2004. 
3. B Chopard, A Dupuis, A Masselot, and P Luthi. Cellular automata and lattice Boltzmann techniques: An approach to model and simulate complex systems. Advances in Complex Systems, 5(2):103-246, 2002.

4. V Coscia, M Delitala, and P Frasca. On the mathematical theory of vehicular traffic flow II: Discrete velocity kinetic models. International Journal of NonLinear Mechanics, 42(3):411-421, 2007.

5. S Dawson, S Chen, and G Doolen. Lattice Boltzmann computations for reaction-diffusion equations. The Journal of Chemical Physics, 98(2):15141523, 1993.

6. CW Gear, TJ Kaper, IG Kevrekidis, and A Zagaris. Projecting to a slow manifold: Singularly perturbed systems and legacy codes. SIAM Journal on Applied Dynamical Systems, 4(3):711-732, 2005.

7. CW Gear and IG Kevrekidis. Projective methods for stiff differential equations: Problems with gaps in their eigenvalue spectrum. SIAM Journal on Scientific Computing, 24(4):1091-1106, 2003.

8. CW Gear and IG Kevrekidis. Constraint-defined manifolds: a legacy code approach to low-dimensional computation. Journal on Scientific Computing, 25(1):17-28, 2005.

9. X He and L-S Luo. Lattice Boltzmann model for the incompressible NavierStokes equation. Journal of Statistical Physics, 88(3):927-944, 1997.

10. M Junk and A Klar. Discretizations for the incompressible Navier-Stokes equations based on the lattice Boltzmann method. SIAM Journal on Scientific Computing, 22(1):1-19, 2000.

11. IG Kevrekidis, CW Gear, JM Hyman, PG Kevrekidis, O Runborg, and C Theodoropoulos. Equation-free, coarse-grained multiscale computation: enabling microscopic simulators to perform system-level tasks. Communications in Mathematical Sciences, 1(4):715-762, 2003.

12. IG Kevrekidis and G Samaey. Equation-free multiscale computation: Algorithms and applications. Annual Review on Physical Chemistry, 60:321-344, 2009.

13. C Li, U Ebert, WJM Brok, and W Hundsdorfer. Spatial coupling of particle and fluid models for streamers: where nonlocality matters. Journal of Physics D: Applied Physics, 41(3):032005, 2008.

14. L-S Luo. Unified theory of lattice Boltzmann models for nonideal gases. Physical Review Letters, 81(8):1618-1621, 1998.

15. HG Othmer and T Hillen. The diffusion limit of transport equations II: Chemotaxis equations. SIAM Journal on Applied Mathematics, 62(4):1222-1250, 2002.

16. Y Qian and S Orszag. Scalings in diffusion-driven reaction $A+B \rightarrow C: \mathrm{Nu}-$ merical simulations by lattice BGK models. Journal of Statistical Physics, 81(1-2):237-253, 1995.

17. L Qiao, R Erban, CT Kelley, and IG Kevrekidis. Spatially distributed stochastic systems: Equation-free and equation-assisted preconditioned computations. The Journal of Chemical Physics, 125:204108, 2006.

18. G Samaey and W Vanroose. An analysis of equivalent operator preconditioning for equation-free Newton-Krylov computations. Technical Report TW537, Department of Computer Science, K.U.Leuven, 2009. Submitted.

19. G Samaey, W Vanroose, D Roose, and IG Kevrekidis. Newton-Krylov solvers for the equation-free computation of coarse traveling waves. Computer Methods in Applied Mechanics and Engineering, 197(43-44):3480-3491, 2008. 
20. S Succi. The lattice Boltzmann equation for fluid dynamics and beyond. Oxford University Press, 2001.

21. C Theodoropoulos, YH Qian, and IG Kevrekidis. Coarse stability and bifurcation analysis using time-steppers: a reaction-diffusion example. Proceedings of the National Academy of Science, 97:9840-9845, 2000.

22. U Trottenberg, C Oosterlee, and A Schuller. Multigrid. Academic Press, 2001.

23. P Van Leemput, W Vanroose, and D Roose. Mesoscale analysis of the equationfree constrained runs initialization scheme. Multiscale Modeling and Simulation, 6(4):1234-1255, 2007.

24. C Vandekerckhove, IG Kevrekidis, and D Roose. An efficient Newton-Krylov implementation of the constrained runs scheme for initializing on a slow manifold. Journal on Scientific Computing, 39(2):167-188, 2009.

25. W Vanroose, G Samaey, and P Van Leemput. Coarse-grained analysis of a lattice Boltzmann model for planar streamer fronts. Technical Report TW479, Department of Computer Science, K.U.Leuven, 2007. 